\title{
Rose Swallow Odibaajimowin imaa Chisaasibiing
}

\author{
Told by Rose Swallow \\ Written by Ruth DyckFehderau \\ Translated by Patricia Ningewance Nadeau
}

Gii-ikwezensiwipan Rose, gaamashi gikino'amaadiiwigamigong gii-ayaasii iwe apii, omishoomisan o-gii-wiijiiwaan wedi Gichi-ziibiing e-gii-izhaawaad ji-naadisabiiwaad. Gii-gichigiizhoopizowag. Animosha' o-gii-odaaba'aawaa' imaa gichi-wiikwedong Hudson Bay gaaizhinikaadeg. Wiin wiin Rose gii-bimidaabaazo, wiinge ishpaagwanagaanig, e-gizhaateg gaye giigizhebaawagak. Gichi-bimiba'idiwag igi animoog, omishoomisan dash bimibatoowan jiigidaabaanaak. Ngoding ako ogijigwaashkwani imaa odaabaanaakong ji-anwebid ajina.

"Inashke!" odinaan oozhisan, "Inashke epiichi-gagiitaawendamowaad igi animoog. Ogikendaanaawaa aaniindi ezhi-naniizaanadaninig, ezhi-bibagizhezigwaag amii dash imaa gaawiin izhaasiiwag. Ogikendaanaawaa. Bizaanigo gidizhaamin ebiwaad nindasabiig gaa-izhigizhiijiwang ziibi, nawach naniizaanizi mikom, wiinawaa dash ogikendaanaawaa aaniindi geizhaasigwaa."

Gaawiin dash wii-ayeko'aasii’ oda'isha, e-gozigonid wiin. Gii-gabaagwaashkwani wiiba, miinawaa e-bimibatood.

Gii-odisaawaad bezhig asabiin, Rose gii-gabaa. Gii-anweshinoog dash igi animoog. Omishoomisan dash gii-naadisabiibani'owan. Gozigwani a'a asab e-bida'anaad giigooya'. Gichigaanjitaa e-zaagijidaabiid ini giigooya' epiichi-gozigoninid. Wewiib ogijigonaa' imaa asabiing ebakitewaad giishpin giiyaabi bimaadizinid. O-gii-dakobinaa' dash imaa, odaabaanaakong o-giiasaa'. Aazha miinawaa boozi Rose. Apane miinawaa godag asabiin enaanzikawaawaad.

Gii-onaagoshininig gii-ayaawaad waakaa'iganensing, o-gii-giizhideboonaa' animoo' omishoomisan, okanan gaye wiiyaas gaye manoomin.

"Weweni gi-daa-ashamaag animoog," odigoon, "Endaso-giizhig, niizhing endaso-giizhig, gaawiin gaye eta gii-odaabiiwaad. Gego wiikaa maanzhidoodawaaken animosh. Weweni doodaw."

Baamaa gaa-ishkwaa-wiisiniwaad animoshag gaa-izhi-onabid wiin, e-wiisinid. 
Gii-niibininig, gii-giigooyikaanig, owiiji'aan ako ookoman Rose e-ozhitoowaad agwaawaanaak. Mitigoonsa' odaabaji'aawaa', gaye ojiitadeyaab. Gii-giizhitoowaad boodawewag imaa, gaa-zhakaakwag mishi odaabajitoonaawaa ji-gichi-biskanesinog. Amii dash imaa ezhiagoonaawaad giigoowa' gaa-ishkwaa-baanizhwaawaad. Gabe-giizhig baasowag. Amii dash bagwaanishing ezhi-gashkiiginaawaad, mashkimoding ezhi-biinawaawaad. Amii dash ezhibimiwanewaad waanikaaning ji-izhi-asanjigowaad. Aki aasaakamig imaa o-gii-atoon ji-onjidakiziwaad igi giigooyag gaa-baasowaad. Gaawiin dash da-onaajishinziiwag niibing gizhideg. Gaa-ishkwaa-ningowaawaad amii ezhi-asaawaad gaa-gozigoninid asinii' ogijiya'ii jiwaanikesigwaa ma'iinganag.

Aya'aawishensa' daabishkoo bineg gaye waaboozoog onisaawaa' gaye gaa-mindidowaad daabishkoo moonzoog adikwag emitosewaad omishoomisan gaye ookoman, maagizhaa gaye enagwaanaawaad ewanii'igewaad. Gii-ishkwaa-naawakwenig ako ookoman Rose owanzaan wiiyaas boodawaanaabikong, bakwezhiganaabo e-ozhitood gemaa naadowensa'. Oodenaang odoondinaawaan ini biisadaawang bakwezhiganan. Gii-baatiinod bakwezhiganike ko.

Mawinzowag ngojigo miinigiizis gii-bimangizod, gii-miinikaag iwe apii. Wiinge ko gichimawinzowag gii-ishkwaa-naawakwenig. Baashkiminisigewan ookoman, gemaa zhiiwibakwezhiganing odasaa'. Amii dash gaye gaa-izhichiged e-gii-baasang ini miinan mashkimoding dash ebiina'ang gaawiin dash wanaadanzinoonan. Amii dash ako gabe-biboon e-ayaawaad niibiwa miijim ge-miijiwaad.

Ngoding gii-maneziwag miijim. Aanawi gii-ozhitoowaad miijim gii-niibininig ge-miijiwaad bibooninig giiyaabi ko gii-jaagisewag. Amii ko wedi minising wiikwedong ezhaanid Rose omishoomisan enaanzikamonid waakon. Nitaawigin imaa ogidaabik. Makadewaa daabishkoo gichi-aniibiish gaa-adaaweng adaawewigamigong izhinaagwan. Wanzigaadeg bizaanigo gi-miijin. Amii gaa-miijiwaad awiyag ji-gawaskadesigwaa mewinzha.

Gii-gichi-anokiiwag mewinzha awiyag ji-bimaadiziwaad, ji-ondinamowaad ge-miijiwaad. Apane go gii

anokiiwag. Gaawiin dash wiikaa awiya gii-ziizibaakwadwaapinesii iwe apii.

Ango-biboon gaa-izhiseg gii-wiijiiwaapan omishoomisan Rose enaadisabiinid, giinamadabi gikino'amaadiiwigamigong e-ganawaabandang gegoon odoonaaganing. Broccoli odizhinikaadaanaawaa igi wemitigoozhii-gikino'amaagewikweg. Gii-inaa ji-miijid gaawiin dash miijim odinendanziin. Oganawaabamigoo'. Giishpin miijisig o-da-bakite'ogoo'. Gemaa gaawiin da-ashamaasii miijim. Ezhi-gashkanzagwaabid enaanaagadawendang gegoon gaa-zaagitood daabishkoo gii-gikino'amawind ji-baakwaa'ishiimod gemaa ji-bakwezhiganiked. Izhi-daawani ebakiindang iweni broccoli. Omaanzhipidaan. Ozhaashaagwandaan. Onoondawaa' godag 
abinoojiizha' e-aa'aagadenid e-gagwe-miijinid iweni broccoli. Nawach maawiin maanzhipogwan owe broccoli apiich wiin waakon inendam. Amii sa gaa-izhi-gidaanawed. Gichi-ayaawiyaan ayaawagwaa abinoojiizhag gaawiikaa nin-ga-ashamaasiig broccoli inendam. Iwe apii gaawiin mashi awiya gii-ziizibaakwadwaapinesii.

Ngoji go 1960 gii-izhisenig biindige gaa-izhi-zaaga'amowaad ikwezensag imaa ishpigikino'amaadiiwigamigong imaa Rouyn-Noranda. Bezhig ikwezens imaa niibawi. Odishpiiginaan ogoodaas, amii dash ezhi-jiita'odizod zhaabonigan e-aabajitood, mashkiki e-miinindizod. Gaaminwendamoshkaagemagak mashkiki odaabajitoon, odinenimaan. Maamakaadendam Rose. Giigiziininjii, amii dash gaa-izhi-maajaad. Gaawiin gegoon odinaasiin. Eniizho-biboonagaak dash Biology gaa-izhinikaadeg gikendaasowining odizhi-bizindawaan gikino'amaagewikwen edazhindaminid ziizibaakwadwaapinewin, e-jiita'odizowaad dash iwe gaa-inaapinewaad. Omikwenimaan ini ikwezensan gaa-gii-waabamaapan e-jiita'odizonid. Amii ngwana nitam e-giiwaabamaad awiyan iwe e-inaapinenid.

Ogii-giizhitoon ogikino'amaagoowin Rose, gii-anokii dash imaa Jizaasabii HBC adaawewigamigong. Ngojigo 1970 gii-izhise iwe apii. Aazha niibiwa gegoon gii-ani-aanjise bimaadiziwin. Gaa-bimibizowaad ogidaagonag gii-ayaawag. Gaawiin noongom animoo' o-giiaabaji'asiwaawaa' ininiwag gii-gii'osewaad gaye gii-bagdawaawaad. Gaawiin noongom o-giigichi-inenimaasiwaawaa' animoo'. Gii-babaamiba'idiwag animoshag miziwe biniskwe. Gaawiin noongom awiyag gii-bimosesiiwag. Gii-izhaawaad adaawewigamigong gii-bimibizowag odaabaanensing. Gaa-mazinaateseg gaye o-gii-ayaanaawaa. Gii-adaawaadegin adaawewigamigong gakina awiya zhemaak gii-adaawe. CBC eta gii-mazinaatese iwe apii, angodiba'igan endaso-giizhig. Amii ko iwe apii gakina awiya gaa-gibitinang wegonen ezhichigewaad e-ando-ganawaabandamowaad gaa-mazinaatesenig.

Bezhig wiisaakodewikwe ngojigo naanimidana daso-biboone Rose o-gii-wiidanokiimaan adaawewigamigong. Apane ko wii-minikwe nibi. Wewiib ako ozhagashkinaanan mazina'iiginoonsan makakong wedi dash gaa-izhi-ayaag gaa-mookojiwang nibi izhaa eminikwed ango-minikwaagan nibi. Biizhaa ajina e-anokiid. Aazha miinawaa wii-minikwe nibi. Amii sa gabe-giizhig iwe ezhichiged e-minikwed nibi gaawiin dash debijii'aabowesii. Gegapii oganoonigoon bezhig owiidanokiimaaganan. Aaniish owaabamigoon bizhishig e-minikwenid nibi gaye gaawiin aapiji waabisiiwan. Odinaan ji-ando-waabamaad mashkikiiwininiwan. Onoondaan e-ayaamagak inaapinewin. Debwe gii-bigiiwe awe ikwe. Odayaan gegoon ge-aabajitood jijiita'odizod. Amii dash gaa-izhichiged endaso-giizhig ejiita'odizod. Amii owe ge-izhichigeyaan wii-bimaadiziyaan odigowaan. Ngoding ako gaawiin deminik iweni gii-jiita'odizod. Apatoo ko imaa gaa-izhi-ozhichigaadeg gaa-makadewaagamig, e-miijid ziizibaakwad. Amii ini miinawaa bezhig gaa-gikenimaad Rose iwe e-inaapinenid. 
Amii noongom iwe inaapinewin e-maajii-dazhinjigaadeg. Niibiwa awiyag iwe inaapinewag. Rose owiijiiwaagana' gaye awiyag gaa-daawaad besho, gaye gichi-ay'aag, gaye odinawemaagana'. Bepezhig Rose oshiimeya' gaye omiseya' iwe inaapinewa'. Bezhig giiozhigwaapine. 1991 gii-izhisenig Rose gii-maadanokii CHR e-inanokiid. Niishtana daso-biboon apane ishkwaawaach gaa-gii-waabamaapan ini ikwezensan ejiita'odizonid. Noongom dash endaso-giizhig owaabamaa' awiya' eziizibaakwadwaapinenid. Endaso-giizis aazha miinawaa awiya wiindamawaa iwe e-inaapined, egoshkwendang dash.

“Gaawiin editawe!" ako ikidowag. "Gaawiin wiikaa nimiijisiin gaa-zhiiwang. Gaawiin iwe nindinaapinesiidog."

Owiindamawaa' idash ako, e-omashkiigomod, bakwezhigan bezhig gegoon gaamaanishkaagemagak. Daabishkoo ziizibaakwad inendaagwan bakwezhigan.

"Nin-gichi-ayaamag o-gii-amwaawaan bakwezhiganan gaawiin dash wiikaa giiziizibaakwadwaapinesiiwag," ikidowag miinawaa.

"Eya,” odinaa'. “O-gii-amwaawaan bakwezhiganan. Wiinge dash gaye gii-gichi-anokiiwag endaso-giizhig. Gaawiin giinawind iwe gidizhi-bimaadizisiimin noongom.”

O-gii-maajii-dazhindaan ziizibaakwadwaapinewin bizinjiganing, ewiindamaaged gaawiin awiya odaa-amwaasiin waabimanoominan gaye opiniin gii-wiisinid. Ziizibaakwad inendaagwanoon ini miijiman. Giishpin ginwesh bimoseyin, gidaa-niisinaan ziizibaakwad gimiskwiiming niizhogon. Giishpin minikweyin ishkodewaabo zhingobiiwaabo zhoominaabo wegonen igo iwe dinookaan, gaawiin onizhishinzinoon ozaam daabishkoo ziizibaakwad inendaagwan. Aapiji niibiwa noongom iwe gii-inaapinewa' gaawiin gii-de-izhisesii ji-wiiji'aad gakina. Ndawaach bezhig miinawaa gii-anokii'aa.

Miinawaa dash bezhig.

Miinawaa bezhig.

Aayaakaw ako Rose gewiin gii-nanaando-gikenindizo ji-ziizibaakwadwaapinegwen. Gaawiin gii-inamanji'osii daabishkoo gaa-inamanji'owaad iwe gaa-inaapinewaad. Niibiwa dash odinawemaagana' o-gii-ayaanaawaa iwe aazha. Gii-ojaanimendam gaye nasine iwe apii giiyaabi gii-nitaa-giiwashkwebiinid onaabeman, ji-boonitoonid. Niibiwa awiyag gii-aakoziwag odazhiikewining. Ginwesh gii-mino-ayaa Rose. Obiidoonan ako iniwe gaa-aabajichigaadegin jigikenjigaadeg. Onanaando-gikenimaan ako onaabeman gaye oniijaanisa'. Amii noongom gaainanjige'aad oniijaanisa' iwe broccoli. 
Aabiding dash 1997 gii-izhisenig gii-nanaando-gikenindizod, gii-ishpaakoshkaani iweni. Amii gewiin iwe ji-inaapined inendam. O-gii-maajii-odaapinaan mashkiki.Nawach gaye giimaajii-babaamose gaye gwayak e-gagwe-inanjiged. Aabiding dash e-gizhaatenig gii-ziigwang 2002 gii-izhisenig, gii-zaaga'am imaa gaa-dananokiid. Aazha tagiin midaaso-diba'iganens giizaaga'amooban aazha dash miinawaa e-inamanji'od. Nanaando-gikenindizo. Wiinge ishpaakoshkaani iweni. Amii sa gewiin Rose Swallow eziizibaakwadwaapined.

Wiinge gichi-ikwanaamo. Bagidanaamo. Apane miinawaa izhaa imaa zaaga'amoowigamigong.

Giiyaabi iwe inanokii Rose imaa Jizaasibiing. Aazha naananiwag iwe gaa-inanokiiwaad. Wiin odazhiikaan e-gikino'amaaged aaniin ge-izhichigeng giishpin ziizibaakwadwaapineyin. Gakina gaa-ayaawaad imaa Jizaasibiing odookishkaagonaawaa iwe inaapinewin. Aazha gaye nawach ani-oshkaadiziwag iwe gaa-inaapinewaad. Amii dash ezhichiged Rose e-wiiji'aad awiya' ji-gojitoowaad ini gitigaanan wiikaa gaa-gii-miijisigwaa gaa-onizhishingin ji-miijinaaniwangin. Izhichige ji-gojipidamowaad jibwaa-adaawewaad adaawewigamigong. Amii gaye ezhichiged ewiindamawaad awiya' ji-mitosenid, gaawiin ji-bimibizosigwaa miziwe. Odinaa' gaye jigagwejiinid. Amii dash enaagwak noongom imaa Jizaasibiing, e-gagwejiiwaad awiyag gagwejiiwigamigong gaye ebimosewaad miikanaang gemaa jiigew ziibiing gii-niibininig, gaye ebabaamaagimosewaad gii-bibooninig. Gaamashi awiyan owaabamaasiin ji-bimibatoonid animoo' gii-odaaba'iwenid daabishkoo gaa-gii-nitaa-izhichigenid omishoomisan.

Daabishkoo gakina ini gaa-gikino'amawaad Rose, amii gewiin ezhichiged e-odaapinaad omashkikiima' endaso-giizhig, gaye e-nanaando-gikendang omiskwiim. Omiijinan gitigaanensan gaye ozaawi-manoominan ogiizizwaan. Gaawiin gaye gagwe-ojaanimendanzii. Giishpin ojaanimendang aapiji wiiba ishpaagoshkaani gaa-aabajitood ji-gikendang ezhisenig omiskwiim. Amii ko ezhi-gichi-babaapagidanaamod nishikaach. Gemaa odagindaan mazina'igan gaazaagitood. Noongom gaye gaawiin minikwesiiwan onaabeman. Noongom oniijaanisa' gaye oozhisa' gaa-ojaanimendami'igod. Ngoding oganawenimaa' oozhisa' giishpin naganaaganiwinid. Aapiji ko ojaanimendam iwe gii-izhisenig.

Amii ko egod onaabeman, "Haaw sa, Rose, aazha gidoojaanimendam gidizhinaagoz. Andobabaamosedaa."

Wawepizowag dash gaye owawepinaawaa' oozhisiwaa' ozaam egisinaanig agwajiing, apane dash e-ando-babaamosewaad miikwanaang, ewaasaagonagaag gaa-izhi-ishpadaasing, e-babaamaagonagiinid oozhisiwaa'. 